\title{
A graph related to the join of subgroups of a finite group
}

\author{
Hadi Ahmadi (*) - BiJan TAeri (**)
}

ABSTRACT - For a finite group $G$ different from a cyclic group of prime power order, we introduce an undirected simple graph $\Delta(G)$ whose vertices are the proper subgroups of $G$ which are not contained in the Frattini subgroup of $G$ and two vertices $H$ and $K$ are joined by an edge if and only if $G=\langle H, K\rangle$. In this paper we study $\Delta(G)$ and show that it is connected and determine the clique and chromatic number of $\Delta(G)$ and obtain bounds for its diameter and girth. We classify finite groups with complete graphs and also classify finite groups with domination number 1 . Also we show that if the independence number of the graph $\Delta(G)$ is at most 7, then $G$ is solvable.

Mathematics Subject Classification (2010). 20D99; 05C25, 05 C83.

KEYWORDs. Graph on groups, subgroup graph, join of subgroups.

\section{Introduction}

There are different ways to associate to a group a certain graph. In this context, it is interesting to ask for the relation between the structure of the group, given in group theoretical terms, and the structure of the graph, given in the language of graph theory.

As a pioneer, Bosák [5], in 1964, defined the graph of subsemigroups of a semigroup. Inspired by his work, Csákány and Pollák [6] in 1969, studied the intersection graph of subgroups of a finite group. Zelinka continued the work on the intersection graph of subgroups of a finite abelian group [12]. Also Shen [11] in 2009, classified finite groups with

(*) Indirizzo dell'A.: Department of Mathematical Sciences, Isfahan University of Technology, 84156-83111, Isfahan, Iran.

E-mail: hadiahmadi@math.iut.ac.ir

(**) Indirizzo dell'A.: Department of Mathematical Sciences, Isfahan University of Technology, 84156-83111, Isfahan, Iran.

E-mail: b.taeri@cc.iut.ac.ir 
disconnected intersection graph of subgroups and solved a problem posed by Csákány and Pollák [6]. More work on graphs associated to subgroups of a group or submodules of a module or ideals of a ring can be found in $[9,4,1,7]$.

Motivated by previous studies on the graph of algebraic structures, for any finite group $G$ different from a cyclic group of prime power order, we define an undirected simple graph $\Delta(G)$ whose vertices are the proper subgroups of $G$ which are not contained in the Frattini subgroup of $G$ and two vertices $H_{1}$ and $H_{2}$ are joined by an edge if $\left\langle H_{1}, H_{2}\right\rangle=G$. Our main goal is to study the connection between the algebraic properties of a group and the graph theoretic properties of the graph associated to it.

Throughout this paper all groups are finite different from a cyclic $p$-group. For a group $G, \pi(G)$ denotes the set of all prime divisors of $|G|$. For $p \in \pi(G)$, the set of all Sylow $p$-subgroups of $G$ is denoted by $\operatorname{Syl}_{p}(G)$ and $n_{p}:=\left|\operatorname{Syl}_{p}(G)\right| . \mathcal{M}(G)$ denotes the set of all maximal subgroups of $G$ and $\Phi(G)$ is the Frattini subgroup of $G$ which is defined as the intersection of all maximal subgroups of $G$.

Let $\Delta$ be a graph with vertex set $V(\Delta)$. The order of $\Delta$ is the number of its vertices, that is $|V(\Delta)|$. The degree of a vertex $v$ denoted by $\operatorname{deg}(v)$ is the number of edges incident to $v$. For distinct adjacent vertices $v_{1}$ and $v_{2}$ we write $v_{1} \sim v_{2}$. If $\Delta$ is connected we denote by $d\left(v_{1}, v_{2}\right)$ the length of a shortest path between $v_{1}$ and $v_{2}$. The diameter of $\Delta$ is defined as $d(\Delta):=\max \left\{d\left(v_{1}, v_{2}\right) \mid v_{1}, v_{2} \in V(\Delta)\right\}$. The girth of $\Delta$, denoted by $g(\Delta)$, is the length of a shortest cycle and a graph with no cycle has infinite girth. A tree is a connected graph which does not contain a cycle.

A star is a tree consisting of one vertex adjacent to all others. A complete graph is a graph in which every pair of distinct vertices are adjacent. A complete graph with $n$ vertices is denoted by $K_{n}$. By a clique in a graph $\Delta$ we mean a complete subgraph of $\Delta$ and the order of a largest clique of $\Delta$ is called the clique number of $\Delta$ and is denoted by $\omega(\Delta)$. For a graph $\Delta$, let $\chi(\Delta)$ denote the chromatic number of $\Delta$, that is the minimum number of colors which can be assigned to the vertices of $\Delta$ such that every two adjacent vertices have different colors. Let $S \subseteq V(\Delta)$, denote by $N_{\Delta}[S]$ the set of vertices in $\Delta$ which are in $S$ or adjacent to a vertex in $S$. If $N_{\Delta}[S]=V(\Delta)$, then $S$ is said to be a dominating set of vertices in $\Delta$. The domination number of a graph $\Delta$, denoted by $\gamma(\Delta)$ is the minimum size of a dominating set of the vertices in $\Delta$. A subset $X$ of the vertices of $\Delta$ is called an independent set if the induced subgraph on $X$ has no edges. The maximum size of an independent set in a graph $\Delta(G)$ is called the independence number of $\Delta$ and is denoted by $\alpha(\Delta)$. All other notations and 
definitions are standard; in the scope of group theory and graph theory; see for instance $[10,3]$.

In this paper, we study some graph properties of the graph $\Delta(G)$. We see that $\Delta(G)$ is always connected with diameter at most 3 and if it contains a cycle, its girth is always 3 or 4 . Also we classify finite groups with complete graphs and also groups whose graphs have domination number 1 . We show that the clique and chromatic number of this graph is equal to the number of maximal subgroups of $G$. Some results about the independence number of $\Delta(G)$ are established. Regularity of $\Delta(G)$, in some partial cases, is dealt with.

\section{Connectivity, clique, chromatic and domination number}

It is easy to see that a group $G$ has a unique maximal subgroup if and only if $G$ is a cyclic $p$-group. We emphasize that in this work $G$ is a finite group different from a cyclic $p$-group where $p$ is a prime number.

DeFinition 2.1. Let $G$ be a group and $\Phi(G)$ denotes its Frattini subgroup. We associate a graph $\Delta(G)$ to $G$ whose vertex set is $\{H<G \mid H \not \Varangle \Phi(G)\}$, consists of proper subgroups $H$ which are not contained in $\Phi(G)$, with two vertices $H_{1}$ and $H_{2}$ adjacent if and only if $G=\left\langle H_{1}, H_{2}\right\rangle$.

REMARK 2.2. Let $G$ be a group and $H \leq G$. We have $H \leq \Phi(G)$ if and only if $\langle H, K\rangle \Varangle G$ for each $1 \neq K \Varangle G$. Because $\Phi(G)$ is exactly the set of non-generator elements of the group. Thus to avoid isolated vertices we consider proper subgroups which are not contained in $\Phi(G)$.

In this section we firstly find the order of this graph and determine its clique and chromatic number. We classify groups with complete graphs. In the sequel we show that this graph is connected and classify groups whose graphs have domination number 1 .

Proposition 2.3. $\Delta(G)$ is an $\left(n_{1}, n_{2}, \ldots, n_{t}\right)$-regular graph for some $n_{i} \geq 1,1 \leq i \leq t$ and $|V(\Delta(G))|=\sum_{i=1}^{t}\left|G: N_{G}\left(H_{i}\right)\right|$.

Proof. The vertex set $V(\Delta(G))$ of the graph, is $G$-invariant under the conjugacy action of $G$. Let $\left[H_{1}\right]_{G},\left[H_{2}\right]_{G}, \ldots,\left[H_{t}\right]_{G}$ be the $G$-orbits of this 
action. Hence $V(\Delta(G))=\cup_{i=1}^{t}\left[H_{i}\right]_{G}$ and so

$$
|V(\Delta(G))|=\sum_{i=1}^{t}\left|\left[H_{i}\right]_{G}\right|=\sum_{i=1}^{t}\left|G: N_{G}\left(H_{i}\right)\right| .
$$

Also for each $i \in\{1,2, \ldots, t\}$ and each $g \in G$, we have $\operatorname{deg}\left(H_{i}\right)=\operatorname{deg}\left(H_{i}^{g}\right)$. Because there exists a bijection $\theta: N_{\Delta}\left[H_{i}\right] \longrightarrow N_{\Delta}\left[H_{i}^{g}\right]$ such that $\theta\left(H_{j}^{x}\right)=H_{j}^{x g}$, where $N_{\Delta}\left[H_{i}\right]=\left\{H_{j}^{x} \in V(\Delta(G)) \mid H_{j}^{x} \sim H_{i}\right\}$ for $x \in G, 1 \leq j \leq t$. Therefore $\left|N_{\Delta}\left[H_{i}\right]\right|=\left|N_{\Delta}\left[H_{i}^{g}\right]\right|$ and $\Delta(G)$ is $\left(n_{1}, n_{2}, \ldots, n_{t}\right)$-regular.

Proposition 2.4. For any group $G$ we have $\chi(\Delta(G))=\omega(\Delta(G))=$ $|\mathcal{M}(G)|$.

Proof. Firstly note that $\omega(\Delta(G)) \leq \chi(\Delta(G))$. Let the vertex $M \in \mathcal{M}(G)$ be colored with a color $C$. Now we can color all subgroups contained in $M$ (if any exist!) with $C$ and since all vertices out of $\mathcal{M}(G)$ are contained in elements of $\mathcal{M}(G)$, we have a coloring for $\Delta(G)$. Hence $\chi(\Delta(G)) \leq|\mathcal{M}(G)|$.

Now note that $\mathcal{M}(G)$ is a clique for $\Delta(G)$ and so $|\mathcal{M}(G)| \leq \omega(\Delta(G))$. This finishes the proof.

THEOREM 2.5. $\Delta(G)$ is complete if and only if $G$ is one of the following types:

(1) $G=P \times P$, where $P$ is a group of prime order.

(2) $G=P \rtimes Q$, where $P$ and $Q$ are groups of prime order.

(3) $G=Q_{8}$, the quaternion group of order 8 .

Proof. If $G$ is one of the types (1), (2), (3), then clearly each vertex of the graph is a maximal subgroup of $G$ and so $\Delta(G)$ is complete.

Conversely, let $\Delta(G)$ be complete. We have $|\mathcal{M}(G)| \geq 2$. Fix $M \in \mathcal{M}(G)$ and $x \in M \backslash \Phi(G)$. Thus $\langle x\rangle \in V(\Delta(G))$. Now $\langle\langle x\rangle, M\rangle=M$, that is $\langle x\rangle$ is not adjacent to $M$ and by completeness of $\Delta(G)$ we have $M=\langle x\rangle$. Let $|x|=p_{1}^{n_{1}} \cdots p_{k}^{n_{k}}$, where the $p_{i}$ 's are distinct prime numbers, $k \geq 1, n_{i} \geq 1$ and $1 \leq i \leq k$. Then there exist elements $x_{i} \in G$ such that $\left|x_{i}\right|=p_{i}^{n_{i}}, 1 \leq i \leq k$ and $M=\langle x\rangle=\left\langle x_{1}\right\rangle \times\left\langle x_{2}\right\rangle \times \cdots \times\left\langle x_{k}\right\rangle$. Obviously there exists $j \in\{1,2, \ldots, k\}$ such that $\left\langle x_{j}\right\rangle \not \Varangle \Phi(G)$. Again by completeness of $\Delta(G)$ we have $M=\left\langle x_{j}\right\rangle$ and so $M$ is a cyclic $p$-group, where $p=p_{j}$. Now two cases hold:

Case 1. $\Phi(G)=1$. If $|\pi(G)|=1$, then $G$ is an elementary abelian $p$-group, that is, $G \cong \frac{G}{\Phi(G)} \cong Z_{p}{ }^{n}$, for some $n \geq 2$. If $n \geq 3$, then clearly 
$\Delta(G)$ is not complete. Hence $n=2$ and so $G \cong Z_{p} \times Z_{p}$ and thus $G$ is of type (1). Now let $|\pi(G)| \geq 2$. Since all maximal subgroups of $G$ are cyclic, all Sylow subgroups of $G$ are also cyclic and by [10, Theorem 10.1.10], $G$ is solvable and splits over $G^{\prime}$, the derived subgroup of $G$. So there exists a subgroup $K$ of $G$ such that $G=G^{\prime} \rtimes K$ and $\left|G^{\prime}\right|$ and $|K|$ are prime numbers. Hence $G$ has type (2).

Case 2. $\Phi(G) \neq 1$. Let $M_{1}, M_{2} \in \mathcal{M}(G)$. There exist prime numbers $p_{1}, p_{2}$ and positive integers $n_{1}, n_{2}$ such that $\left|M_{1}\right|=p_{1}^{n_{1}},\left|M_{2}\right|=p_{2}^{n_{2}}$. Since $\Phi(G) \neq 1, p_{1}=p_{2}$. This means that all maximal subgroups of $G$ are cyclic $p$-group, for some prime $p$. Hence $|G|=p^{n}$, for some $n \geq 1$ and all maximal subgroups of $G$ are cyclic. Now by [10, Theorem 5.3.4] which classifies finite $p$-groups with a cyclic maximal subgroup, up to isomorphism, $G$ has one of the following types:

(i) $Z_{p^{n}}$

(ii) $Z_{p^{n-1}} \times Z_{p}$

(iii) $\left\langle x, a \mid x^{p}=1=a^{p^{n-1}}, a^{x}=a^{1+p^{n-2}}\right\rangle ; n \geq 3$

(iv) The dihedral group $D_{2^{n}}, n \geq 3$, of order $2^{n}$

(v) Generalized quaternion group $Q_{2^{n}}=\langle x, y| x^{2^{n-1}}=1, y^{2}=x^{2^{n-2}}$, $\left.x^{y}=x^{-1}\right\rangle ; n \geq 3$

(vi) Semidihedral group $S D_{2^{n}}=\left\langle x, a \mid x^{2}=1=a^{2^{n-1}}, a^{x}=a^{2^{n-2}-1}\right\rangle$; $n \geq 3$.

Recall that all groups in the paper are different from a cyclic group of prime power order, so the case (i) cannot happen.

Suppose that (ii) holds. If $n \geq 3$, then $G$ has a non-cyclic maximal subgroup and so $n=2$. Thus $G$ has type (1).

Now suppose that (iii) holds. Then clearly $\langle x\rangle$ and $\left\langle x, a^{p^{n-2}}\right\rangle$ are two distinct vertices of $\Delta(G)$ which are not adjacent, contradicting the completeness of $A(G)$ so this case does not happen.

Similarly the cases (iv) and (vi) cannot happen, as we can find two distinct vertices which are not adjacent.

Finally suppose that (v) holds. If $n=3$, then $G=Q_{8}$ and $\Delta(G)=K_{3}$ so $\Delta(G)$ is complete. When $n \geq 4$, it suffices to consider two distinct vertices $\langle y\rangle$ and $\left\langle y, x^{2^{n-3}}\right\rangle$ of $\Delta(G)$ which are not adjacent again contradicting the completeness of $\Delta(G)$. Hence in this case the only possibility is $Q_{8}$.

LEMma 2.6. For a group $G$ we have the following properties:

(1) $\Delta(G)$ is a connected graph with $d(\Delta(G)) \leq 3$. 
(2) If $\Delta(G)$ contains a cycle, then $3 \leq g(\Delta(G)) \leq 4$.

(3) For an arbitrary normal subgroup $N$ of $G$ with $N \leq \Phi(G)$; we have $\alpha(\Delta(G)) \geq \alpha\left(\Delta\left(\frac{G}{N}\right)\right)$.

Proof. (1) Let $H_{1}, H_{2}$ be arbitrary vertices of $V(\Delta(G))$. Since $H_{i} \not \leq \Phi(G), i=1,2$, there exist $M_{i} \in \mathcal{M}(G)$ such that $H_{i} M_{i}$ (may be $\left.M_{1}=M_{2}\right)$. Therefore $\left\langle H_{1}, M_{1}\right\rangle=G=\left\langle H_{2}, M_{2}\right\rangle$ and $H_{1} \sim M_{1} \sim M_{2} \sim H_{2}$, which means that $\Delta(G)$ is connected and $d(\Delta(G)) \leq 3$.

(2) Let $M_{1}, M_{2} \in \mathcal{M}(G)$ and take $z \in G \backslash\left(M_{1} \cup M_{2}\right)$. If $\langle z\rangle \lesseqgtr G$, then we have a triangle in $\Delta(G)$ with vertices $\left\{M_{1},\langle z\rangle, M_{2}\right\}$, hence $g(\Delta(G))=3$. Thus let $G=\langle z\rangle$ and $|z|=p_{1}{ }^{n_{1}} \cdots p_{k}{ }^{n_{k}}$, where the $p_{i}$ 's are distinct prime numbers $k \geq 2$ and $n_{i} \geq 1$ for $1 \leq i \leq k$. Hence

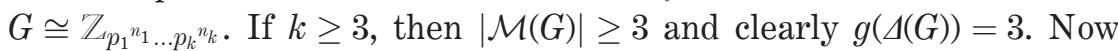
let $k=2$. If $n_{1}=1$ or $n_{2}=1$, then $\Delta(G)$ is a star graph and so does not contain a cycle; a contradiction. Thus $n_{1} \geq 2$ and $n_{2} \geq 2$, from which it follows easily that $g(\Delta(G))=4$.

(3) This is straightforward.

Proposition 2.7. (1) If $\alpha(\Delta(G)) \leq 7$, then $G$ is a solvable group.

(2) If $G$ is a nilpotent group such that $\Delta(G)$ is non-complete, then

$$
\alpha(\Delta(G)) \geq \max \left\{\left|G: N_{G}(H)\right| \mid H \in V(\Delta(G))\right\}+1 .
$$

Proof. (1) Let $\bar{G}:=\frac{G}{\Phi(G)}$. By (3) of Lemma 2.6, we have $\alpha(\Delta(\bar{G})) \leq$ $\alpha(\Delta(G))$. Hence $\alpha(\Delta(\bar{G})) \leq 7$. Thus each maximal subgroup $\bar{M}$ of $\bar{G}$ contains at most 7 proper non-trivial subgroup. Therefore if $|\pi(\bar{M})| \geq 2$ and $\bar{M}$ is nonnilpotent, by an inspection we can show that each Sylow subgroup of $\bar{M}$ is cyclic. Hence $\bar{M}$ is supersolvable, thus $\bar{G}$ is solvable (see [10, Theorems 10.1.10 and 10.3.4]). Since $\Phi(G)$ is solvable, $G$ is solvable.

(2) Suppose, if possible, that there exists a maximal subgroup of $G$ of prime order $p$. Then by nilpotency of $G,|G|=p q$, where $q$ is a prime. Since $G$ is non-cyclic of prime power order, we have $G \cong Z_{p} \times Z_{q}$, which implies that $\Delta(G)$ is complete, a contradiction. Therefore the order of every maximal subgroup of $G$ is not prime.

Now let $H \in V(\Delta(G))$. There exists a maximal subgroup $M$ of $G$ such that $H \leq M$. Since $G$ is nilpotent, every maximal subgroup $M$ of $G$ is normal. Therefore $H^{g} \leq M$ for each $g \in G$. Hence $[H]_{G} \cup\{M\}$ is an independent set of $\Delta(G)$. Since $H$ is arbitrary, the result follows. 
REMARK 2.8. The converse of Proposition 2.7 (1), does not hold, as it is shown by the elementary abelian group $Z_{p}^{m}$, where $m \geq 3, p \geq 7$.

Recall that, by [10, Theorem 9.2.1], every finite solvable group $G$ has a Sylow basis, namely a set of mutually permutable Sylow subgroups, one for each prime dividing the group order.

Theorem 2.9. $\quad \gamma(\Delta(G))=1$ if and only if $G$ is solvable with a Sylow basis $\left\{P_{1}, P_{2}\right\}$ such that $P_{1}$ is a cyclic maximal subgroup of $G$ and $\Phi(G)$ is a maximal subgroup of $P_{1}$, or $G$ is a $p$-group of order $p^{n}$, where $n$ is a positive integer and $G$ is isomorphic to one of the groups:

(1) $Z_{p} \times Z_{p^{n-1}}, p$ is a prime number

(2) $\left\langle x, a \mid x^{p}=1=a^{p^{n-1}}, a^{x}=a^{1+p^{n-2}}\right\rangle$; $p$ is a prime number and $n \geq 3$

(3) The dihedral group $D_{2^{n}}, n \geq 3$, of order $2^{n}$

(4) Generalized quaternion group $Q_{2^{n}}, n \geq 3$

(5) Semidihedral group $S D_{2^{n}}=\left\langle x, a \mid x^{2}=1=a^{2^{n-1}}, a^{x}=a^{2^{n-2}-1}\right\rangle$; $n \geq 3$.

Proof. First let $G$ be a solvable group with Sylow basis $\left\{P_{1}, P_{2}\right\}$, where $P_{1}$ is a maximal subgroup of $G$ and $\Phi(G)$ is a maximal subgroup of $P_{1}$. We show that $\left\{P_{1}\right\}$ is a dominating set for $\Delta(G)$. Suppose that $H \in$ $V(\Delta(G)) \backslash\left\{P_{1}\right\}$. If $H<P_{1}$, then $H \Phi(G) \leq P_{1}$ and maximality of $\Phi(G)$ in $P_{1}$ implies that $H \Phi(G)=P_{1}$. Since $G=P_{1} P_{2}=H \Phi(G) P_{2}=H P_{2}$, and thus $H=P_{1}$ a contradiction. Therefore $H \not \subset P_{1}$ and so $\left\langle H, P_{1}\right\rangle=G$, that is $H \sim P_{1}$.

Now let $G$ be one of the types (1), (2), (3), (4), (5), then $\bar{G}:=\frac{G}{\Phi(G)} \cong Z_{p}^{m}$, $m \geq 2$. By [10, Theorem 5.3.4] $G$ has a cyclic maximal subgroup $A$. Let $\bar{A}:=\frac{A}{\Phi(G)}$, which is a cyclic maximal subgroup of $\bar{G}$, so $\bar{A}=\langle\bar{a}\rangle$ for some $\bar{a} \in \bar{G}$ and $|\bar{A}|=p^{m-1}$. Since $\bar{G}$ is an elementary abelian $p$-group, $m=2$. Thus $|\Phi(G)|=p^{n-2}$. If $K \in V(\Delta(G)) \backslash\{A\}$, then $K \not \subset A$, because $A$ is cyclic. Therefore $\langle K, A\rangle=G$ and so $K \sim A$. Thus if $G$ has one of the types (1)-(5), then $\gamma(\Delta(G))=1$.

Conversely suppose that $\gamma(\Delta(G))=1$ and $D=\{A\}$ is a dominating set for $\Delta(G)$. We claim that $A$ is a cyclic maximal subgroup of $G$. If not, then there exists a maximal subgroup $M$ of $G$ such that $A \Varangle M$ and $A$ is not adjacent to $M$, which is a contradiction. Hence $A$ is a maximal subgroup of $G$. Now take $a \in A \backslash \Phi(G)$. Then the vertex $\langle a\rangle$ is not ad- 
jacent to $A$ and so $A=\langle a\rangle$ and the claim is proved. Clearly $\Phi(G)$ is a maximal subgroup of $A$. Therefore $G$ is a solvable group and $A$ is of primary index (see [10, Exercise 10.5.7 and 5.4.3-(iii)]). Suppose that $|G: A|=p^{n}$, where $p$ is prime and $n \geq 1$ and $P$ is a Sylow $p$-subgroup of $G$. If $G$ is a $p$-group, then by [10, Theorem 5.3.4] $G$ is one of the types (1), (2), (3), (4) and (5). Therefore suppose that $G$ is not a $p$-group. Since $G$ is a solvable group, it posseses a Sylow basis $\left\{P_{1}, P_{2}, \ldots, P_{l}, P\right\}, l \geq 1$.

Without loss of generality we can assume that $P_{i} \subseteq A, 1 \leq i \leq l$, (see [8]). If $P_{1} \Varangle A$, then $P_{1}$ is not adjacent with $A$, a contradiction. Thus $A=P_{1}$, that is $\{A, P\}$ is a Sylow basis of $G$ as desired. This completes the proof.

\section{Bipartiteness and regularity}

In this section we firstly investigate $\Delta(G)$, when it is a cycle or when it is bipartite. Then we classify groups whose graphs are $r$-regular for $r \in\{3,4\}$.

Proposition 3.1. (1) $\Delta(G)$ is a cycle if and only if $G \cong Q_{8}$ or $Z_{p_{1}^{2} p_{2}^{2}}$ or $Z_{2} \times Z_{2}$, where $p_{1}, p_{2}$ are distinct prime numbers.

(2) $\Delta(G)$ is bipartite if and only if $G \cong Z_{p_{1}^{n_{1}} p_{2}^{n_{2}}}$ where $n_{i} \geq 1, i \in\{1,2\}$.

Proof. (1) If $G$ is one of the groups $Q_{8}$ or $Z_{2} \times Z_{2}$, then $\Delta(G)=K_{3}$. Also if $G \cong Z_{p_{1}^{2} p_{2}^{2}}$, then $\Delta(G)=C_{4}$ where $C_{n}$ denotes the cycle of length $n, n \geq 1$.

Conversely let $\Delta(G)$ be a cycle. By Lemma 2.6, $\Delta(G)$ is connected and $d(\Delta(G)) \leq 3$. Hence $\Delta(G) \in\left\{C_{3}, C_{4}, C_{5}, C_{6}, C_{7}\right\}$. Suppose, if possible, that $\Delta(G)=C_{5}$ and $V(\Delta(G))=\left\{H_{1}, H_{2}, H_{3}, H_{4}, H_{5}\right\} \quad$ and $\quad \Delta(G)$ is: $H_{1} \sim H_{2} \sim H_{3} \sim H_{4} \sim H_{5} \sim H_{1}$. It is easy to see that $|\mathcal{M}(G)|=2$, say $\mathcal{M}(G)=\left\{H_{1}, H_{2}\right\}$. Thus $H_{4} \leq \Phi(G)$ so $H_{4} \notin V(\Delta(G))$, a contradiction. Similarly $\Delta(G) \in\left\{C_{6}, C_{7}\right\}$, is impossible.

Therefore $\Delta(G) \in\left\{C_{3}, C_{4}\right\}$. First let $\Delta(G)=C_{3}$ so $\Delta(G)$ is complete and by Theorem 2.5, $G$ is isomorphic to one of the following: $Z_{p} \times Z_{p}$ or $Z_{p} \times Z_{q}$ or $Q_{8}$. Clearly $V(\Delta(G))=\mathcal{M}(G)$. If $G \cong Z_{p} \times Z_{p}$, then $\Delta(G)=K_{p+1}$ and so $p=2$. Hence $G \cong Z_{2} \times Z_{2}$. Now suppose, if possible, that $G \cong Z_{p} \times Z_{q}$. We can assume that $p \neq q$ and so we have $V(\Delta(G))=\operatorname{Syl}_{p}(G) \cup \operatorname{Syl}_{q}(G)$. Thus $|V(\Delta(G))|=n_{p}+n_{q}=1+p>1+2=3$ a contradiction. Therefore this case is impossible. 
Now let $\Delta(G)=C_{4}$. Then $|\mathcal{M}(G)|=2$ and $G=\langle g\rangle$ is a cyclic group. Suppose that $|g|=p_{1}^{n_{1}} p_{2}^{n_{2}} \cdots p_{k}^{n_{k}}$, where the $p_{i}$ 's are distinct primes and $n_{i} \geq 1,1 \leq i \leq k$. Since $|\mathcal{M}(G)|=2$ we conclude that $k=2$ and

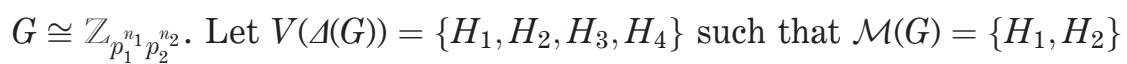
and $\Delta(G)$ is the cycle: $H_{1} \sim H_{2} \sim H_{3} \sim H_{4} \sim H_{1}$. If $n_{1} \geq 3$ or $n_{2} \geq 3$, then $\Delta(G)$ contains $K_{1,3}$ as a subgraph, a contradiction. Therefore $\left(n_{1}, n_{2}\right) \in$ $\{(1,1),(1,2),(2,1),(2,2)\}$. If $\left(n_{1}, n_{2}\right) \in\{(1,1),(1,2),(2,1)\}$, then $|V(\Delta(G))| \in$ $\{2,3\}$. contradicting $|V(\Delta(G))|=4$. Thus $\left(n_{1}, n_{2}\right)=(2,2)$, that is $G \cong Z_{p_{1}^{2} p_{2}^{2}}$ and the proof is complete.

(2) If $\Delta(G)$ is bipartite, it contains no odd cycle and this implies that $|\mathcal{M}(G)|=2$. Hence $G$ is cyclic and the proof of (1) gives the result. The converse is obvious.

Proposition 3.2. (1) $\Delta(G)$ is 3-regular if and only if $G$ has one of the types $Z_{p_{1}^{3} p_{2}}, Z_{3} \times Z_{3}$ or $S_{3}$, where $p_{1}$ and $p_{2}$ are distinct primes and $S_{3}$ is the symmetric group on three symbols.

(2) $\Delta(G)$ is 4 -regular if and only if $G \cong Z_{p_{1}^{4} p_{2}^{4}}$.

Proof. (1) If $G \cong Z_{p_{1}^{3} p_{2}^{3}}$, then one can see that $\Delta(G)$ has exactly six vertices and $G$ has exactly two maximal subgroups with no common neighbors. Thus $\Delta(G)$ is 3 -regular. In fact $\Delta(G)=K_{3,3}$. If $G=S_{3}$ or $G=Z_{3} \times Z_{3}$, then $\Delta(G)=K_{4}$.

Conversely let $\Delta(G)$ be 3 -regular. Thus $|\mathcal{M}(G)| \leq 4$. First let $|\mathcal{M}(G)|=2$. Therefore $G$ is cyclic with two distinct prime divisors $p_{1}, p_{2}$. Note that the maximal subgroups have no common neighbors. So $|V(\Delta(G))|=6$ and thus $G \cong Z_{p_{1}^{3} p_{2}^{3}}$. Now suppose that $|\mathcal{M}(G)|=4$. Then $\Delta(G)=K_{4}$ and by Theorem 2.5, $G \cong P \times Q$ or $G=P \times P$, where $P$ and $Q$ are groups of prime order. If $G=P \times P$, then $\Delta(G)=K_{p+1}$ and so $p=3$,

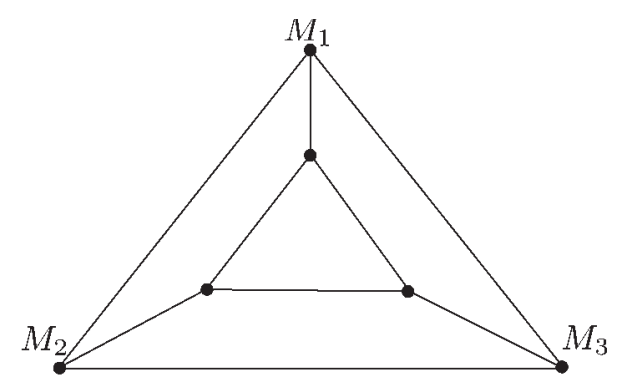

Fig. 1. 
that is $G \cong Z_{3} \times Z_{3}$. If $G \cong P \times Q$, then $V(\Delta(G))=\operatorname{Syl}_{p}(G) \cup \operatorname{Syl}_{q}(G)$ and obviously we have $|P|=3$ and $|Q|=2$. Thus $G \cong S_{3}$.

Finally let $|\mathcal{M}(G)|=3$. By [2, Lemma 1] $G$ is a cyclic group of order $p^{n} q^{m} r^{k}$, where $p, q, r$ are distinct primes and $n, m, k \geq 1$ or $G$ is a 2-group. In both cases one can easily see that $|V(\Delta(G))|=6$ and any two of the maximal subgroups of $G$ have no common neighbors as shown in Figure 1. Now note that $\Phi(G)=M_{i} \cap M_{j}$, for each $M_{i}, M_{j} \in$ $\mathcal{M}(G), 1 \leq i, j \leq 3$. So if $H$ is a non-maximal vertex of $\Delta(G)$, then $H \leq \Phi(G)$, which is a contradiction. Thus this case does not hold and the proof is complete.

(2) Let $G \cong Z_{p_{1}^{4} p_{2}^{4}}$. Then $|V(\Delta(G))|=8$ and $|\mathcal{M}(G)|=2$. So $\Delta(G)$ has six non-maximal vertex and clearly it is 4 -regular. In fact $\Delta(G)=K_{4,4}$.

Conversely if $\Delta(G)$ is 4 -regular, then $|\mathcal{M}(G)| \leq 5$. First let $|\mathcal{M}(G)|=5$. So $\triangle(G)=K_{5}$ and by Theorem 2.5 , we have $G \cong P \times P$ or $G \cong P \rtimes Q$ where $P$ and $Q$ are groups of prime orders. By an easy inspection we can see that neither case can happen. We claim that if $|\mathcal{M}(G)|=4$, then $\Delta(G)$ cannot be $4-$ regular and this case also does not hold. In fact if $\Delta(G)$ is 4 -regular, then $G$ has a non-maximal subgroup $H$ which contained in exactly one maximal vertex of $\Delta(G)$, as $\Phi(G)=M_{i} \cap M_{j}$, for each $M_{i}, M_{j} \in \mathcal{M}(G), 1 \leq i, j \leq 4$. Since $\operatorname{deg}(H)=4, \Delta(G)$ has another non-maximal vertex different from $H$, say $K$. Similarly $K$ is contained in exactly one maximal vertex of $\Delta(G)$. This shows that there exists a maximal vertex of $\Delta(G)$ whose degree is greater than 4 , which is a contradiction. Thus the claim is proved.

Now let $|\mathcal{M}(G)|=3$. Similar to the proof of (1), we have $|V(\Delta(G))|=6$ and so any two maximal subgroups of $G$ have a common neighbour and every maximal subgroup of $G$ contains exactly one vertex of the graph which is shown in Figure 2.

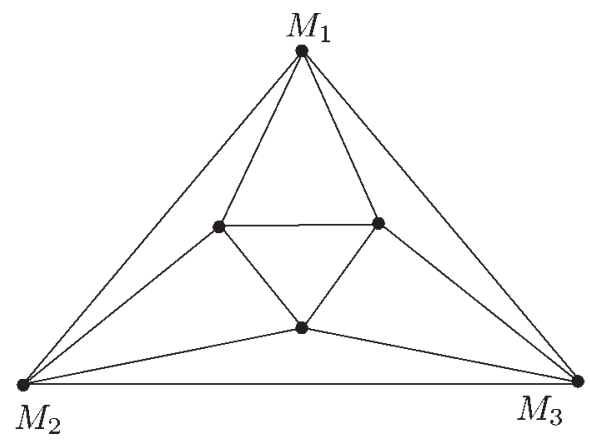

Fig. 2. 
Again by [2, Lemma 1] $G$ is a cyclic group of order $p^{n} q^{m} r^{k}$, where $p, q, r$ are distinct primes and $n, m, k \geq 1$ or $G$ is a 2-group such that $\frac{G}{\Phi(G)} \cong Z_{2} \times Z_{2}$. If $G$ is of the first type, then obviously each maximal subgroup of $G$ contains more than one vertex of $\Delta(G)$, which is a contradiction. If $G$ is of the second type, then each maximal subgroup of $G$ is cyclic. To see this suppose that $H$ is a non-maximal vertex of the graph. Then there is a maximal subgroup $M$ of $G$ such that $H \Varangle M$. Now take $x \in M \backslash(H \cup \Phi(G))$. Then $\langle x\rangle \in V(\Delta(G))$ and $\langle x\rangle \leq M$. But exactly one vertex of $\Delta(G)$ is contained in $M$. Hence $M=\langle x\rangle$. Therefore $\Phi(G)$ is a maximal subgroup of $H$ and also $H$ is maximal in $M$ and so we have:

$$
|G: \Phi(G)|=|G: M||M: H||H: \Phi(G)|=2^{3},
$$

which is a contradiction.

Finally let $|\mathcal{M}(G)|=2$. Then $G$ is cyclic with two distinct prime divisors $p_{1}, p_{2}$. Since $\Delta(G)$ is 4 -regular, every maximal subgroup of $G$ contains exactly three non-maximal vertex of the graph. Also maximal subgroups of $G$ have no common neighbor. Therefore $G \cong Z_{p_{1}^{4} p_{2}^{4}}$ and $\Delta(G)=K_{4,4}$, which completes the proof.

Acknowledgments. The authors wish to thank the referee for his/her valuable remarks and suggestions.

\section{REFERENCES}

[1] S. Akbari - H. A. Tavallayee - S. Khalashi Ghezelahmad, Intersection graph of submodules of a module, Journal of Algebra and its Applications, 1 (2012), Article No. 1250019.

[2] A. ASHRAFI, On the number of minimal and maximal subgroups of a finite group, J. Sci. Univ. Tehran, 3 (1998), pp. 15-20.

[3] Balakrishnan - K. Ranganathan, A text book of graph theory, SpringerVerlag New York Heidelberg Berlin, 1991.

[4] A. Ballester-Bolinches - J. Cossey - R. Esteban-Romero, On a graph related to permutability in finite groups, Annali di Matematica, 189 (2010), pp. 567-570.

[5] J. Bosák, The graphs of semigroups in theory of graphs and Applications, Academic Press New York, (1964), pp. 119-125.

[6] B. CSÁKÁNy - G. Pollák, The graph of subgroups of a finite group, Czechoslovak Math. J., 19 (1969), pp. 241-247.

[7] I. Chakrabarty - S. Ghosh - T. K. Mukherjee - M. K. Sen, Intersection graphs of ideals of rings, Discrete Math., 309 (2009), pp. 5381-5392.

[8] P. HALL, On the Sylow systems of a soluble group, Proceedings of the London Mathematical Society, 43 (1937), pp. 316-323. 
[9] M. Herzog - P. Longobardi - M. MAJ, On a graph related to maximal subgroups of a group, Bull. Aust. Math. Soc., 81 (2010), pp. 317-328.

[10] D. J. S. Robinson, A course in the theory of groups, Springer-Verlag New York Heidelberg Berlin, 1996.

[11] R. SHEN, Intersection graphs of subgroups of finite groups, Czechoslovak Math. J., 60 (2010), pp. 945-950.

[12] B. ZELINKA, Intersection graphs of finite abelian groups, Czechoslovak Math. J., 25 (1975), pp. 171-174.

Manoscritto pervenuto in redazione il 26 Maggio 2013. 